Proceedings of the

International Geometry Center

Vol. 13, no. 4 (2020) pp. 1-23

\title{
A survey of the homotopy nilpotency and co-nilpotency
}

\author{
Marek Golasiński
}

\begin{abstract}
We review known and state some new results on the homotopy nilpotency and co-nilpotency of spaces. Next, we take up the systematic study of the homotopy nilpotency of homogeneous spaces $G / K$ for a Lie group $G$ and its closed subgroup $K<G$. The homotopy nilpotency of the loop spaces $\Omega\left(G_{n, m}(\mathbb{K})\right), \Omega\left(F_{n ; n_{1}, \ldots, n_{k}}(\mathbb{K})\right)$, and $\Omega\left(V_{n, m}(\mathbb{K})\right)$ of Grassmann $G_{n, m}(\mathbb{K})$, flag $F_{n ; n_{1}, \ldots, n_{k}}(\mathbb{K})$ and Stiefel $V_{n, m}(\mathbb{K})$ manifolds for $\mathbb{K}=\mathbb{R}, \mathbb{C}$, the field of reals or complex numbers and $\mathbb{H}$, the skew $\mathbb{R}$-algebra of quaternions is studied.
\end{abstract}

Анотація. В роботі наведено деякі відомі та встановлено нові результати про гомотопічну нільпотентністі та ко-нільпотентність просторів. Зокрема, ми систематично досліджуємо гомотопічну нільпотентність однорідних просторів $G / K$ для групи Лі $G$ та її замкненої підгрупи $K<G$, та просторів петель $\Omega\left(G_{n, m}(\mathbb{K})\right), \Omega\left(F_{n ; n_{1}, \ldots, n_{k}}(\mathbb{K})\right)$ та $\Omega\left(V_{n, m}(\mathbb{K})\right)$ многовидів Грасмана $G_{n, m}(\mathbb{K})$, многовидів прапорів $F_{n ; n_{1}, \ldots, n_{k}}(\mathbb{K})$ та многовидів Штіфеля $V_{n, m}(\mathbb{K})$ для полів $\mathbb{K}=\mathbb{R}, \mathbb{C}$ та тіла кватерніонів $\mathbb{H}$.

2010 Mathematics Subject Classification: Primary: 55P15, secondary: 20F18, 55P45, $55 \mathrm{P} 60$.

Keywords: classical Lie group, co- $H$-space, flag manifold, (oriented) flat product, (oriented) Grassmann manifold, homogeneous space, homology decomposition, homotopy nilpotency (co-nilpotency) class, $H$-fibration, $n$-fold commutator (co-commutator) map, $H$-space, loop space, Morava $K$-theory, $p$-localization, Postnikov system, projective space, sphere, Stiefel manifold, suspension space, smash product.

Ключові слова: класичні групи Лі, ко- $H$-простір, многовид прапорів, (орієнтовний) плоский добуток, (орієнтований) многовид Грасмана, однорідний простір, розклад груп гомологій, клас гомотопічної нільпотентності (ко-нільпотентності), $\quad H$ розшарування, відображення $n$-кратного комутування (ко-комутування), $H$-простір, простір петель, $K$-теорія Морави, $p$-локалізація, система Постнікова, проєктивний простір, сфера, многовид Штіфеля, надбудова, смеш-добуток.

DOI: http://dx.doi.org/10.15673/tmgc.v13i4.1750 


\section{INTRODUCTION}

In the group theory, if we consider only nilpotent groups, the nilpotency class is the one which measures a distance from commutativity. Already G. Whitehead [38] had the insight that the (J.H.C.) Whitehead products satisfy identities which reflect commutator identities for groups. Berstein and Ganea [5] adopt the nilpotency to $H$-spaces as follows. Let $X$ be an $H$-space, $\varphi_{X, 1}=\operatorname{id}_{X}$, and $\varphi_{X, 2}: X^{2} \rightarrow X$ the commutator map. Put $\varphi_{X, n+1}=\varphi_{X, 1} \circ\left(\operatorname{id}_{X} \times \varphi_{X, n}\right)$ for $(n+1)$-fold commutator map of $X$ with $n \geqslant 2$. An $H$-space $X$ is called homotopy nilpotent of class $n$ if $\varphi_{X, n+1} \simeq *$, is null homotopic but $\varphi_{X, n}$ is not [5]. In this case, we write nil $X=n$. Dually, it is adopted the co-nilpotency to co- $H$-spaces.

Then, Berstein and Ganea [5] introduced a concept of the homotopy nilpotency (resp. homotopy co-nilpotency) of a pointed space by means of its loop space (resp. suspension space). Its fibrewise version has been studied by James [24]. In particular, the $m$-iterated Samelson products vanish in the loop space $\Omega(X)$, or equivalently, that the $m$-iterated Whitehead products (resp. $\cup$-products) vanish in $X$ provided $m>\operatorname{nil} \Omega(X)$ (resp. $m>\operatorname{co-nil} \Sigma(X))$.

The homotopy nilpotency classes nil $X$ of associative $H$-spaces $X$ has been extensively studied as well as their homotopy commutativity. Work of Hopkins [22] drew renewed attention to such problems by relating this classical nilpotency notion with the nilpotence theorem of Devinatz, Hopkins, and Smith [11]. In particular, Hopkins [22] made a big progress by giving cohomological criteria for homotopy associative finite $H$-spaces to be homotopy nilpotent. For example, he showed that if a homotopy associative finite $H$-space has no torsion in the integral homology, then it is homotopy nilpotent. Later, Rao [33] showed that the converse of the above criterion is true in the case of groups $\operatorname{Spin}(n)$ and $\operatorname{SO}(n)$. Eventually, Yagita [40] proved that, when $G$ is a compact, simply connected Lie group, its $p$-localization $G_{(p)}$ is homotopy nilpotent if and only if $G$ has no torsion in the integral homology. Finally, Rao [34] showed that a connected compact Lie group is homotopy nilpotent if and only if it has no torsion in homology.

Crabb, Sutherland and Zhang in [10] got surprisingly low bounds for the homotopy nilpotency class of gauge groups when the bundles are stages of in the Milnor construction of the classifying space for a compact Lie group $G$, even when $G$ itself is not homotopy nilpotent. Furthermore, they have proved an equivariant version of Hoppkin's result for unitary groups. Although many results on the homotopy nilpotency are obtained as above and others, e.g., [27,37], the homotopy nilpotency classes have not been determined in almost all cases. 
Before describing our purpose, it is worthwhile to mention a few recent contributions along these lines. Berger and Bourn [4] study nilpotency in the context of exact Mal'tsev categories taking central extensions as the primitive notion. This yields a nilpotency tower which is analysed from the perspective of Goodwillie's functor calculus. They show in particular that the reflection into the subcategory of $n$-nilpotent objects is the universal endofunctor of degree $n$ if and only if every $n$-nilpotent object is $n$-folded. In the special context of a semi-Abelian category, an object is $n$-folded precisely when its Higgins commutator of length $(n+1)$ vanishes.

Biedermann and Dwyer [7] study the connection between the Goodwillie tower of the identity and the lower central series of the loop group on connected spaces. They define homotopy nilpotent groups as homotopy algebras over certain simplicial algebraic theories. This notion interpolates between infinite loop spaces and loop spaces, but backwards. Then, the relation to ordinary nilpotent groups is studied. It is proved that $n$-excisive functors of the form $\Omega(F)$ factor over the category of homotopy $n$-nilpotent groups.

Kaji and Kishimoto [26] consider the problem: how far from being homotopy commutative is a loop space having the homotopy type of the $p$ completion of a product of finite numbers of spheres? They determine the homotopy nilpotency of those loop spaces as an answer to this problem.

We aim to review in this survey paper known and state some new results on the homotopy nilpotency and co-nilpotency of spaces. In Section 1, we set stages for developments to come. This introductory section is devoted to a general discussion and establishes notations on the homotopy nilpotency (resp. co-nilpotency) of $H$-spaces (resp. co- $H$-spaces) used in the rest of the paper.

Sections 2 and 3 make use of $[5,14,17]$ to present know and state some new results on the homotopy nilpotency and co-nilpotency of spaces. Finally, Section 4, based mainly on [16], takes up the systematic study of the homotopy nilpotency of homogeneous spaces $G / K$ for a Lie group $G$ and its closed subgroup $K<G$. The homotopy nilpotency of the loop spaces $\Omega\left(G_{n, m}(\mathbb{K})\right), \Omega\left(F_{n ; n_{1}, \ldots, n_{k}}(\mathbb{K})\right)$, and $\Omega\left(V_{n, m}(\mathbb{K})\right)$ of Grassmann $G_{n, m}(\mathbb{K})$, flag $F_{n ; n_{1}, \ldots, n_{k}}(\mathbb{K})$ and Stiefel $V_{n, m}(\mathbb{K})$ manifolds for $\mathbb{K}=\mathbb{R}, \mathbb{C}$, the field of reals or complex numbers and $\mathbb{H}$, the skew $\mathbb{R}$-algebra of quaternions is studied. In particular, the homotopy nilpotency nil $\Omega\left(\mathbb{H} P^{n}\right)<\infty$ is shown for the quaternionic projective space $\mathbb{H} P^{n}$ with $n \geqslant 1$ which does not appear in the literature known to the author. 


\section{Prerequisites}

All spaces and maps in this note are assumed to be connected and based with the homotopy type of $C W$-complexes unless we assume otherwise. We also do not distinguish notationally between a continuous map and its homotopy class. We write $\Omega(X)$ (resp. $\Sigma(X))$ for the loop (resp. suspension) space on a space $X$ and $[X, Y]$ for the set of homopoty classes of maps $X \rightarrow Y$.

Given a space $X$, we use the customary notations $X \vee X$ and $X \wedge X$ for the wedge and the smash square of $X$, respectively.

Let us recall that an $H$-space is a pair $(X, \mu)$, where $X$ is a space and $\mu: X \times X \rightarrow X$ is a map such that the diagram

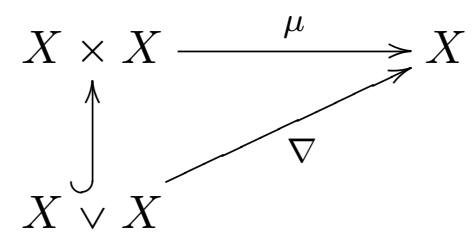

commutes up to homotopy, where $\nabla: X \vee X \rightarrow X$ is the folding map. We call $\mu$ a multiplication or an $H$-structure for $X$. Two examples of $H$-spaces come in mind: topological groups and the spaces $\Omega(X)$ of loops on $X$. In the sequel, we identify an $H$-space $(X, \mu)$ with the space $X$.

Dually, recall that a co-H-space is a pair $\left(X^{\prime}, \mu^{\prime}\right)$, where $X^{\prime}$ is a space and $\mu^{\prime}: X^{\prime} \rightarrow X^{\prime} \vee X^{\prime}$ is a map such that the diagram

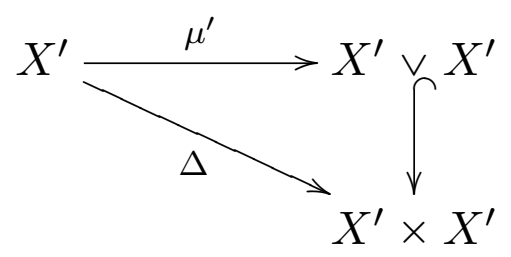

commutes up to homotopy, where $\Delta: X^{\prime} \rightarrow X^{\prime} \times X^{\prime}$ is the diagonal map.

We call $\mu^{\prime}$ a co-multiplication or a co-H-structure for $X^{\prime}$. Two examples of co- $H$-spaces come in mind: Moore space $M(A, n)$ of type $(A, n)$ for an Abelain group $A$ and $n \geqslant 2$, and the suspension spaces $\Sigma(X)$. In the sequel, we identify an co- $H$-space $\left(X^{\prime}, \mu^{\prime}\right)$ with the space $X^{\prime}$.

An $H$-space $X$ (resp. a co- $H$-space $X^{\prime}$ ) is called a group-like space (resp. co-group-like space) if $X$ (resp. $X^{\prime}$ ) satisfies all the axioms of groups (resp. co-group) up to homotopy. Recall that a homotopy associative an $\mathrm{H}$ $C W$-complex (resp. a 1-connected homotopy co-associative a co- $H-C W$ complex) always has a homotopy inverse (resp. co-inverse). More precisely, according to [42, 1.3.2. Corollary] (see also [2, Proposition 8.4.4]), we have:

Proposition 1.1. (1) If $X$ is a homotopy associative $H$-CW-complex then $X$ is a group-like space. 
(2) If $X^{\prime}$ is a 1-connected homotopy co-associative co-H-CW-complex then $X^{\prime}$ is a co-group-like space.

From now on, we assume that any $H$-space $X$ (resp. co- $H$-space $X^{\prime}$ ) is group-like (resp. co-group like).

Given spaces $X_{1}, \ldots, X_{n}$, we use the customary notations $X_{1} \times \cdots \times X_{n}$ and $X_{1} \vee \cdots \vee X_{n} \subseteq X_{1} \times \cdots \times X_{n}$ for their Cartesian and wedge products of $X_{1}, \ldots, X_{n}$, respectively. We also write $X_{1} \wedge \cdots \wedge X_{n}$ for their smash product.

Let $f_{m}:\left(X_{m}, \star_{m}\right) \rightarrow\left(Y_{m}, *_{m}\right)$ be continuous maps of pointed topological spaces for $m=1, \ldots, n$. The map

$f_{1} \times \cdots \times f_{n}:\left(X_{1} \times \cdots \times X_{n},\left(\star_{1}, \ldots, \star_{n}\right)\right) \rightarrow\left(Y_{1} \times \cdots \times Y_{n},\left(*_{1}, \ldots, *_{n}\right)\right)$

sends the point $\left(x_{1}, \ldots, x_{n}\right) \in X_{1} \times \cdots \times X_{n}$ to $\left(f_{1}\left(x_{1}\right), \ldots, f_{n}\left(x_{n}\right)\right)$. We write

$$
\begin{gathered}
j_{n}(X): X_{1} \vee \cdots \vee X_{n} \rightarrow X_{1} \times \cdots \times X_{n}, \\
f_{1} \vee \cdots \vee f_{n}: X_{1} \vee \cdots \vee X_{n} \rightarrow Y_{1} \vee \cdots \vee Y_{n}
\end{gathered}
$$

for the inclusion map and the restriction of $f_{1} \times \cdots \times f_{n}$ to the wedge product $X_{1} \vee \cdots \vee X_{n}$, respectively. If $X_{m}=X$ and $f_{m}=f$ for $m=1, \ldots, n$ then we write

$$
\begin{gathered}
X^{n}=X_{1} \times \cdots \times X_{n}, \quad{ }^{n} X=X_{1} \vee \cdots \vee X_{n}, \quad X^{\wedge n}=X_{1} \wedge \cdots \wedge X_{n}, \\
f^{n}=f_{1} \times \cdots \times f_{n}, \quad{ }^{n} f=f_{1} \vee \cdots \vee f_{n} .
\end{gathered}
$$

The diagonal map $\Delta: X \rightarrow X^{n}$ is defined by $\Delta(x)=(x, \ldots, x)$ and the folding map $\nabla:{ }^{n} X \rightarrow X$ by

$$
\nabla\left(\star_{1}, \ldots, \star_{m-1}, x, \star_{m+1}, \ldots, \star_{n}\right)=x
$$

for $x \in X$ and $m=1, \ldots, n$. The identity map of a space $X$ involved is consistently denoted by $\iota_{X}$.

Given an $H$-space $X$ and a co- $H$-space $X^{\prime}$, the functors $[-, X]$ and $\left[X^{\prime},-\right]$ takes its values in the category of groups. One may then ask when those functors take their values in various subcategories of groups. For example, $X$ (resp. $X^{\prime}$ ) is homotopy commutative (resp. co-commutative) if and only if $[Y, X]$ (resp. $\left.\left[X^{\prime}, Y\right]\right)$ is Abelian for all $Y$.

For an $H$-space $X$, we write

$$
\varphi_{1, X}=\iota_{X}, \quad \varphi_{2, X}: X^{2} \rightarrow X
$$

for the basic commutator map and

$$
\varphi_{n+1, X}=\varphi_{2, X} \circ\left(\varphi_{n, X} \times \iota_{X}\right)
$$

for $n \geqslant 2$. 
The nilpotency class nil $X$ of an $H$-space $X$ is the least integer $n \geqslant 0$ for which the map $\varphi_{n+1, X} \simeq *$ is nullhomotopic and call the homotopy associative $H$-space $X$ homotopy nilpotent. If no such, integer exists, we put nil $X=\infty$. Thus, nil $X=0$ if and only if $X$ is contractible and, as is easily seen, nil $X \leqslant 1$ if and only if $X$ is homotopy commutative.

The set $\pi_{0}(X)$ of all path-components of an $H$-space $X$ is known to be a group. The following result is easy to prove:

Lemma 1.2. If $X$ is an $H$-space and the path component of the base-point $\star \in X$ is contractible, then nil $\pi_{0}(X)=\operatorname{nil} X$.

Dually, for a co- $H$-space $X^{\prime}$ we write

$$
\psi_{1, X^{\prime}}=\iota_{X^{\prime}}, \quad \psi_{2, X^{\prime}}: X^{\prime} \rightarrow{ }^{2} X^{\prime}
$$

for the basic co-commutator map and

$$
\psi_{n+1, X^{\prime}}=\left(\psi_{n, X^{\prime}} \vee \iota_{X}\right) \circ \psi_{2, X^{\prime}}
$$

for $n \geqslant 2$. The co-nilpotency class co-nil $X^{\prime}$ of a co- $H$-space is the least integer $n \geqslant 0$ for which the map $\psi_{n+1, X^{\prime}} \simeq *$ is nullhomotopic and call the homotopy co-associative co- $H$-space $X$ homotopy co-nilpotent. If no such integer exists, we put co-nil $X^{\prime}=\infty$.

The definitions of the nilpotency and co-nilpotency classes may be extended to maps. The nilpotency class nil $f$ of an $H$-map $f: X_{1} \rightarrow X_{2}$ is the least integer $n \geqslant 0$ for which the map $f \circ \varphi_{n+1, X}: X_{1}^{n+1} \rightarrow X_{2}$ is nullhomotopic. If no such integer exists, we put nil $f=\infty$.

The co-nilpotency class co-nil $g$ of a co- $H$-map $g: X_{1}^{\prime} \rightarrow X_{2}^{\prime}$ is the least integer $n \geqslant 0$ for which the map $\psi_{n+1, X_{2}^{\prime}} \circ g: X_{1}^{\prime} \rightarrow{ }^{n+1} X_{2}^{\prime}$ is nullhomotopic. If no such integer exists, we put co-nil $g=\infty$.

In the sequel we need:

Lemma 1.3. (1) If $X$ is an $H$-space then the composite map

$$
{ }^{n} X \stackrel{j_{n}(X)}{\longrightarrow} X^{n} \stackrel{\varphi_{n, X}}{\longrightarrow} X
$$

is nullhomotopic.

(2) If $X^{\prime}$ is a co-H-space then the composite map

$$
X^{\prime n} \stackrel{j_{n}\left(X^{\prime}\right)}{\longrightarrow}{ }^{n} X^{\prime} \stackrel{\psi_{n, X^{\prime}}}{\longrightarrow} X^{\prime}
$$

is nullhomotopic.

Notice that $X^{\wedge n}$ is the homotopy cofiber (the $n$-th smash power of $X$ ) of $j_{n}(X):{ }^{n} X \rightarrow X^{n}$ and write ${ }^{b n} X^{\prime}$ for the homotopy fiber (the $n$-th flat power of $X^{\prime}$ ) of $j_{n}\left(X^{\prime}\right):{ }^{n} X^{\prime} \rightarrow X^{\prime n}$. Then, the result above implies an 
existence of maps

$$
\bar{\varphi}_{n, X}: X^{\wedge n} \rightarrow X \quad \text { and } \quad \bar{\psi}_{n, X^{\prime}}: X^{\prime} \rightarrow{ }^{b n} X^{\prime} .
$$

Recall that ${ }^{b n} X^{\prime} \simeq{ }^{* n} \Omega\left(X^{\prime}\right)$, the $n$-th join power of $\Omega\left(X^{\prime}\right)$. Then, by [31, Corollary, p. 135], we have

$$
{ }^{b n} X^{\prime} \simeq \Sigma^{n-1} \Omega\left(X^{\prime}\right)^{\wedge n} .
$$

Notice that [1, Corollary 4.3], [16, Lemma 1.2], and [19, Corollary 1] lead to:

Lemma 1.4. (1) If $X$ is a group-like $C W$-space and $Y$ a finite dimensional $C W$-complex with $\operatorname{dim} Y=n$ then the group $[Y, X]$ is nilpotent with the nilpotency class at most $n$.

(2) If $X^{\prime}$ is a 1-connected co-group-like $C W$-space and $Y$ is a $C W$ complex with an n-stage Postnikov system then the group $\left[X^{\prime}, Y\right]$ is nilpotent with the nilpotency class at most $n$.

Proof. (1) First, recall that given an $H$-CW-space $X$, in view of [25], all its $m$-th Postnikov stages $P_{m} X$ are also an $H$-space and the canonical maps $X \rightarrow P_{m} X$ are $H$-maps for $m \geqslant 1$. Since the map $X \rightarrow P_{n} X$ is an $(n-1)$-homotopy equivalence and $Y$ is a $C W$-complex with $\operatorname{dim} Y=n$, there is an isomorphism $[Y, X] \approx\left[Y, P_{n} X\right]$ of groups determined by the map $X \rightarrow P_{n} X$. Then, the map

$$
\bar{\varphi}_{P_{n} X, n+1}\left(f_{1} \wedge \cdots \wedge f_{n+1}\right): Y^{\wedge(n+1)} \rightarrow P_{n} X
$$

is homotopy trivial for any maps $f_{1}, \ldots, f_{n+1}: Y \rightarrow P_{n} X$ since the space $Y^{\wedge}(n+1)$ is $n$-connected. Consequently, nil $[Y, X]=\operatorname{nil}\left[Y, P_{n} X\right] \leqslant n$.

(2) We dualize mutatis mutandis the proof of (1) as follows. Given a 1connected co- $H$ - $C W$-space $X^{\prime}$, in view of [1, Corollary 4.3] and [19, Corollary 1], all its $m$-th homology decomposition stages $P_{m}^{\prime} X^{\prime}$ are also co- $H$ spaces and the canonical maps $P_{m}^{\prime} X^{\prime} \rightarrow X^{\prime}$ are co- $H$-maps for $m \geqslant 2$.

Next, the map $P_{n}^{\prime} X^{\prime} \rightarrow X^{\prime}$ is an $(n-1)$-homology equivalence, so it is an $(n-1)$-cohomology equivalence as well. Since, $X^{\prime}$ is a $C W$-space, we deduce that induced maps

$$
\begin{aligned}
{\left[X^{\prime}, K\left(\pi_{m}(Y), m\right)\right] \approx H^{m}(} & \left.X^{\prime}, \pi_{m}(Y)\right) \rightarrow \\
& \rightarrow\left[P_{n}^{\prime} X^{\prime}, K\left(\pi_{m}(Y), m\right)\right] \approx H^{m}\left(X^{\prime}, \pi_{m}(Y)\right)
\end{aligned}
$$

are isomorphisms for $m \leqslant n-1$, where $K(\pi, m)$ is the Eileberg-MacLane space of type $(\pi, m)$. Hence, for a $C W$-complex $Y$ with an $n$-stage Postnikov system, there is an isomorphism $\left[P_{n}^{\prime} X^{\prime}, Y\right] \approx\left[X^{\prime}, Y\right]$ of groups determined by the canonical map $P_{n}^{\prime} X^{\prime} \rightarrow X^{\prime}$. Then, the map

$$
\bar{\psi}_{P_{n}^{\prime} X^{\prime}, n+1}\left(g_{1} b \cdots b g_{n+1}\right): P_{n}^{\prime} X^{\prime} \rightarrow^{b(n+1)} Y
$$


is homotopy trivial for any maps $g_{1}, \ldots, g_{n+1}: P_{n}^{\prime}\left(X^{\prime}\right) \rightarrow Y$ since the space

$$
{ }^{b(n+1)} Y \simeq \Sigma^{n-1} \Omega(Y)^{\wedge n}
$$

is $(n-1)$-connected. Consequently, nil $\left[X^{\prime}, Y\right]=$ nil $\left[P_{n}^{\prime} X^{\prime}, Y\right] \leqslant n$ and the proof follows.

Next, any $C W$-complex $Y$ can be expressed as

$$
Y=\lim _{\rightarrow} Y_{\alpha}
$$

where $Y_{\alpha}$ are finite $C W$-complexes. Given a group-like space $X$, this leads to the short exact sequence

$$
1 \rightarrow \lim _{\leftarrow}^{1}\left[\Sigma Y_{\alpha}, X\right] \longrightarrow[Y, X] \longrightarrow \lim _{\leftarrow}\left[Y_{\alpha}, X\right] \rightarrow 1,
$$

where the group $\lim _{\leftarrow}\left[Y_{\alpha}, X\right]$, by means of Lemma 1.4(1), is visibly pronilpotent. By the proof of [22, Proposition 1.2], the intersection

$$
\Gamma_{d} \cap\left(\lim _{\leftarrow}^{1}\left[\Sigma Y_{\alpha}, X\right]\right)=0
$$

for $\operatorname{dim} X=d<\infty$, where $\Gamma_{d}$ stands for the $d$-th member of the lower central series of the group $[Y, X]$. This leads, by means of $[22$, Proposition 1.1], to pro-nilpotency of the group $[Y, X]$ provided $X$ is a finite group-like $C W$-space.

Dually, given a co-group like $C W$-space $X^{\prime}$ with $\operatorname{dim} X^{\prime}=d<\infty$, we get that the group isomorphism $\left[X^{\prime}, Y\right] \approx\left[X^{\prime}, P_{d} Y\right]$ for any $C W$-complex $Y$ for the $d$-th Postnikov stage $P_{d} Y$ of $Y$.

Thus, in view of Lemma 1.4, we obtain:

Proposition 1.5. (1) If $X$ is a finite group-like $C W$-space then the group $[Y, X]$ is pro-nilpotent for any $C W$-complex $Y$.

(2) If $X^{\prime}$ is a 1-connected finite dimensional co-group-like $C W$-space then the group $\left[X^{\prime}, Y\right]$ is nilpotent for any $C W$-complex $Y$.

Notice that there is the short exact sequence

$$
1 \rightarrow \lim _{\leftarrow}^{1}\left[X^{\prime}, \Omega P_{n} Y\right] \longrightarrow\left[X^{\prime}, Y\right] \longrightarrow \lim _{\leftarrow}\left[X^{\prime}, P_{n} Y\right] \rightarrow 1
$$

for any co-group-like space $X^{\prime}$ and a $C W$-space $Y$. If $\operatorname{dim} X^{\prime}=d<\infty$ then there is a group isomorphism $\lim _{\leftarrow}\left[X^{\prime}, P_{n} Y\right] \approx\left[X^{\prime}, P_{d} Y\right]$ and the group isomorphism $\left[X^{\prime}, Y\right] \approx\left[X^{\prime}, P_{d} Y\right]$ leads to the byproduct

$$
\lim _{\leftarrow}^{1}\left[X^{\prime}, \Omega P_{n} Y\right]=0 .
$$

On the other hand, this result is a consequence of the Mittag-Leffler condition as well. 
It is well known that the quotient map $X^{n} \rightarrow X^{\wedge n}$ has a right homotopy inverse after suspending, and the fact that $X$ is an $H$-space means that the suspension map $[Y, X] \rightarrow[\Sigma(Y), \Sigma(X)]$ is a monomorphism for any space $Y$. A dual remark might be stated on co- $H$-spaces as well. Thus, we may state:

Proposition 1.6. Let $X$ be an $H$-space and $X^{\prime}$ a co-H-space. Then:

(1) $\varphi_{n, X} \simeq *$ if and only if $\bar{\varphi}_{n, X} \simeq *$;

(2) $\psi_{n, X^{\prime}} \simeq *$ if and only if $\bar{\psi}_{n, X} \simeq *$.

We point out that details of the proof of Proposition 1.6(1) are presented in [17, Theorem 2.1]. Whereas, the proof of Proposition 1.6(2) follows from the dual version of [17, Theorem 2.1].

Then, [5, 2.7. Theorem] and Proposition 1.6 lead to:

Theorem 1.7. (1) If $X$ is an $H$-space then

nil $X=\sup _{m}$ nil $\left[X^{m}, X\right]=\sup _{m}$ nil $\left[X^{\wedge m}, X\right]=\sup _{Y}$ nil $[Y, X]$,

where $m$ ranges over all integers and $Y$ over all topological spaces.

(2) If $X^{\prime}$ is a co-H-space then

co-nil $X^{\prime}=\sup _{m}$ nil $\left[X^{\prime},{ }^{m} X^{\prime}\right]=\sup _{m} \operatorname{nil}\left[X^{\prime},{ }^{b n} X^{\prime}\right]=\sup _{Y} \operatorname{nil}\left[X^{\prime}, Y\right]$, where $m$ ranges over all integers and $Y$ over all topological spaces.

Furthermore, in view of [42, Lemma 2.6.1], we may state:

Corollary 1.8. (1) A connected $H$-space $X$ is homotopy nilpotent if and only if the functor $[-, X]$ on the category of all spaces is nilpotent group valued.

(2) A connected co-H-space $X^{\prime}$ is homotopy nilpotent if and only if the functor $\left[X^{\prime},-\right]$ on the category of all spaces is nilpotent group valued.

Proof. (1) Certainly, the homotopy nilpotency of a connected associative $H$-space $X$ implies that the functor $[-, X]$ on the category of all pointed spaces is nilpotent group valued.

Now, suppose that the functor $[-, X]$ is nilpotent group valued and nil $\left[\prod_{1}^{\infty} X, X\right]<n$. Then, for the projection map $\prod_{1}^{\infty} X \rightarrow X^{n}$ on the first $n$ factors, the composite map

$$
\prod_{1}^{\infty} X \rightarrow X^{n} \stackrel{\varphi_{n, X}}{\longrightarrow} X
$$

is null-homotopic. Since, the projection $\prod_{1}^{\infty} X \rightarrow X^{n}$ is a retraction, we deduce that the map $\varphi_{n, X}: X^{n} \rightarrow X$ is also null-homotopic. 
(2) It goes mutatis mutandis dually to (1) and the proof is complete.

Example 1.9. (1) Certainly, nil $\mathbb{S}^{1}=1$ and, in view of [30], we have $\operatorname{nil} \mathbb{S}^{3}=3$

(2) co-nil $\mathbb{S}^{1}=\sup _{m}$ nil $\left[\mathbb{S}^{1},{ }^{m} \mathbb{S}^{1}\right]=\infty$ since $\left[\mathbb{S}^{1},{ }^{m} \mathbb{S}^{1}\right]$ is the free group with rank $m \geqslant 1$;

(3) recall that by [6], for any co-associative co- $H$-space $X^{\prime}$ there exists a homotopy equivalence $X^{\prime} \simeq Y \vee S$, where $Y$ is 1-connected and $S$ is a wedge of circles or a point. Now, if $X^{\prime}$ is non-simply connected then:

(a) for $S=\mathbb{S}^{1}$, the obvious surjection $\left[X^{\prime}, X^{\prime} \vee X^{\prime}\right] \rightarrow\left[\mathbb{S}^{1}, \mathbb{S}^{1} \vee \mathbb{S}^{1}\right]$ which is a group homomorphism implies that the group $\left[X^{\prime}, X^{\prime} \vee X^{\prime}\right]$ is non-nilpotent;

(b) $S={ }^{n} \mathbb{S}^{1}$ with $n \geqslant 2$ the obvious surjection $\left[X^{\prime}, X^{\prime}\right] \rightarrow\left[\mathbb{S}^{1}, S\right]$ which is a group homomorphism implies that the group $\left[X^{\prime}, X^{\prime}\right]$ is non-nilpotent.

Consequently, by Theorem 1.7(2), we have co-nil $X^{\prime}=\infty$ for any nonsimply connected co-associative co- $H$-space $X^{\prime}$.

\section{Properties of the homotopy nilpotency}

We make mainly use of $[5,14,17]$ to present know and state some new results on the homotopy nilpotency of loop spaces.

With any based space $X$, we associate the integer nil $\Omega(X)$ (respectively co-nil $\Sigma(X)$ ) called the homotopy nilpotency (resp. co-nilpotency) class of $X$.

Evidently, nil $\pi_{1}(X) \leqslant \operatorname{nil} \Omega(X)$. We give an extension of this result involving Whitehead products, generally denoted by $\left[\alpha_{1}, \alpha_{2}\right] \in \pi_{m_{1}+m_{2}-1}(X)$ if $\alpha_{i} \in \pi_{m_{i}}(X)$ for $m_{i} \geqslant 1$ with $i=1,2$.

We define $(n+1)$-fold Whitehead products $\left[\alpha_{1}, \ldots, \alpha_{n+1}\right]$ as

$$
\left[\left[\alpha_{1}, \ldots, \alpha_{n}\right], \alpha_{n+1}\right]
$$

if $\alpha_{i} \in \pi_{m_{i}}(X)$ for $m_{i} \geqslant 1$ with $i=1, \ldots, n+1$ agreeing that, for $n=0$, $[\alpha]=\alpha$.

Recall that W-length $X$, the Whitehead length of a space $X$ is the least integer $n \geqslant 0$ such that $\left[\alpha_{1}, \ldots, \alpha_{n+1}\right]=0$ for all $\alpha_{i} \in \pi_{m_{i}}(X), m_{i} \geqslant 1$. If no such integer exists, we put W-length $X=\infty$.

Then, according to [5, 4.6. Theorem], we have:

Theorem 2.1. W-length $X \leqslant \operatorname{nil} \Omega(X)$.

Example 2.2. It is well-known that

$$
\text { W-length } \mathbb{S}^{n}=\operatorname{nil} \mathbb{S}^{n}= \begin{cases}3, & \text { for } n \text { even with } n \neq 2, \\ 2, & \text { for } n \text { odd with } n \neq 1,3,7 \text { or } n=2, \\ 1, & \text { for } n=1,3,7\end{cases}
$$


Given a path-connected space $X$, the action of $\pi_{1}(X)$ on $\pi_{n}(X)$ for $n \geqslant 1$ may be written in terms of Whitehead products. Then, by Theorem 2.1, the space $X$ is nilpotent if $\Omega(X)$ is homotopy nilpotent.

We now proceed to find upper bounds for nil $\Omega(X)$. First, let $X$ be a connected aspherical $C W$-complex. Then $\pi_{m}(X)=0$ for all $m>1$. Therefore, Lemma 1.2 yields:

Proposition 2.3. If $X$ is a connected aspherical $C W$-complex, then

$$
\operatorname{nil} \pi_{1}(X)=\operatorname{nil} \Omega(X) .
$$

For further reference, we state the easily proved:

Lemma 2.4. $\operatorname{nil}\left(X_{1} \times X_{2}\right)=\max \left\{\operatorname{nil} X_{1}\right.$, nil $\left.X_{2}\right\}$.

Recall that a space $X$ is said to dominate a space $Y$ provided there are continuous mappings $f: X \rightarrow Y$ and $g: Y \rightarrow X$ such that $f \circ g$ is homotopic to the identity mapping $\iota_{Y}$.

Next, in view of [14], the co-category, co-cat $X$, of a topological space $X$ is a (possibly infinite) strictly positive integer as given by: co-cat $X=1$ if and only if $X$ is contractible.

Let $n \geqslant 1$ be given and suppose the phrase co-cat $Y=m$ has been defined for any space $Y$ and all integers $m$ satisfying $1 \leqslant m \leqslant n$; then, co-cat $X=n+1$ provided:

(i) co-cat $X \neq m$ for any in satisfying $1 \leqslant m \leqslant n$, and

(ii) there exists a fibration $Q \rightarrow Y \rightarrow B$ such that $Q$ dominates $X$ and co-cat $Y=n$.

If co-cat $X \neq n$ for all $n \geqslant 1$, we put co-cat $X=\infty$.

Then, by [14, Theorem 2.12], we have:

Theorem 2.5. nil $\Omega(X) \leqslant \operatorname{co-cat} X-1$.

We notice that applying the principal refinement of the Postnikov system of a path-connected nilpotent space, [5, 4.11. Theorem] yields:

Theorem 2.6. Let $X$ be a path-connected nilpotent $C W$-complex. Suppose the invariants $k^{n+2}(X)$ of a Postnikov system for $X$ are trivial for almost all values of $n$. Then nil $\Omega(X)<\infty$.

In particular, if $X$ is a path-connected nilpotent $C W$-complex with a finite Postnikov system then $\operatorname{nil} \Omega(X)<\infty$.

Corollary 2.7. Let $X$ be a path-connected nilpotent $C W$-complex.

(i) If $\pi_{n}(X)=0$ for almost all values of $n$, then $\operatorname{nil} \Omega(X)<\infty$; 
(ii) if the Postnikov invariants $k^{n+2}(X)$ vanish for almost all values of $n$ then $\mathrm{W}$-length $X<\infty$.

Consequently, Theorems 2.1 and 2.5 yield

$$
\text { W-length } X \leqslant \operatorname{nil} \Omega(X) \leqslant \operatorname{co-cat} X-1 \text {. }
$$

Notice that Theorem 2.5 leads to the following dual version of Whitehead's [39] result (cf. Lemma 1.4(2)):

Corollary 2.8. If $Y$ is a space with co-cat $Y \leqslant n$ then the nilpotency nil $\left[X^{\prime}, Y\right] \leqslant n-1$ for any co-group-like space $X^{\prime}$.

Proof. Recall that, by [15, Theorem 2.2], for any co-group-like space $X^{\prime}$ there is a decomposition via co- $H$-map (up to homotopy) of the identity map $\iota_{X^{\prime}}$ as $X^{\prime} \rightarrow \Sigma \Omega X^{\prime} \rightarrow X^{\prime}$. Hence, we may consider the group $\left[X^{\prime}, Y\right]$ as a subgroup of $\left[\Sigma \Omega X^{\prime}, Y\right.$. From Theorem 2.5, we deduce that the nilpotency of $\left[X^{\prime}, Y\right]$ is less than or equal to nil $\Omega(Y) \leqslant$ co-cat $Y-1=n-1$ and the proof follows.

In particular, we deduce that nil $[X, \Omega(Y)]=\operatorname{nil}[\Sigma(X), Y] \leqslant n-1$ for any spaces $X, Y$ provided co-cat $Y \leqslant n$.

Example 2.9. (1) It is well-known that

$$
\operatorname{nil} \Omega\left(\mathbb{S}^{n}\right)= \begin{cases}3, & \text { for } n \text { even with } n \neq 2, \\ 2, & \text { for } n \text { odd with } n \neq 1,3,7 \text { or } n=2, \\ 1, & \text { for } n=1,3,7\end{cases}
$$

(2) For the wedge $\mathbb{S}^{m} \vee \mathbb{S}^{n}$ of two spheres with $m, n \geqslant 2$, there is an iterated nontrivial Whitehead product of any weight. Therefore, we conclude that

$$
\operatorname{nil} \Omega\left(\mathbb{S}^{m} \vee \mathbb{S}^{n}\right)=\infty
$$

Hence, not every space $\Omega(X)$ is homotopy nilpotent if $X$ is nilpotent or even simply connected.

We close this section by reviewing some recent works related to the subject above.

The Lusternik-Schnirelmann category of a space $X$, LS-cat $X$ is the minimal number of open sets needed to cover $X$ which are contractible in $X$. This was originally defined for manifolds, and is a lower bound for the number of critical points of a function on $X$. The definition was broadened to arbitrary spaces, and later definitions include an inductive version, ind LS-cat by Ganea [14], and symmetric version, sym LS-cat, by Hopkins [21]. 
Co-category is much less well understood than category. The first attempt do define co-category was made by Ganea [14]. Different possible definitions of co-category exist depending on which feature of the classical LS-category is dualized.

Hopkins [21] presents new formulations of category and co-category closer in spirit to the original definition of category [14]. One byproduct of these formulations is a new characterization of iterated loop spaces and a dual characterization of iterated suspensions.

Eldred [12] uses constructions in Goodwillie's calculus of homotopy functors to reformulate Hopkins's definition [21] of symmetric LS-cocat and applies to spaces determined by functors associated to reduced homotopy endofunctors of spaces. Her result is concluded by the following inequalities

W-length $X \leqslant \operatorname{nil} X \leqslant$ ind LS-cocat $X \leqslant \operatorname{sym}$ LS-cocat $X$.

Hovey [23] introduces a new definition which of a co-category. Cocategory has some advantages over the previous two definitions. It dualizes Whitehead's definition of category, so it is defined by a map making a suitable diagram commute.

Murillo and Viruel [29] present a new approach to the Lusternik-Schnirelmann co-category of a space. This approach is based on a dual of the Whitehead definition of category. Using this new definition they are able to prove all the classical properties satisfied by the original Ganea's concept of co-category.

Yau [41] introduces Clapp-Puppe type generalized Lusternik-Schnirelmann co-category in a Quillen model category and establishes some of their basic properties and give various characterizations of them. As the application of these characterizations, it is shown that the generalized co-category is invariant under Quillen modelization equivalences. In particular, generalized co-category of spaces and simplicial sets coincide. Another application of these characterizations is to define and study rational co-category.

\section{Properties of the homotopy CO-Nilpotency}

We make mainly use of $[5,14]$ to present know and state some new results on the homotopy co-nilpotency of suspension spaces.

First, notice that a dual version of $[5,4.11$. Theorem $]$ might be stated for homology decomposition (see e.g., [20, Chapter 8]) as follows:

Theorem 3.1. Let $X$ be a 1-connected $C W$-complex with a finitely generated integral singular homology group $H_{2}(X)$. Suppose the dual Postnikov invariants $k_{n+2}^{\prime}(X)$ of a homology decomposition of $X$ are trivial for almost all values of $n$. Then nil $\Sigma(X)<\infty$. 
In particular, if $X$ is a 1-connected $C W$-complex with a finitely generated integral singular homology group $\mathrm{H}_{2}(X)$ and a finite homology decomposition system then co-nil $\Sigma(X)<\infty$.

Now, recall that $\cup$-length $Y$ of a space $Y$ is the least integer $n \geqslant 0$ such that, for any commutative coefficient field $K$, the cup product of any $(n+1)$ singular cohomology $H^{*}(Y, K)$ classes of positive dimension vanishes. If no such integer exists, we put $\cup$-length $Y=\infty$.

Then, [5, 5.8. Theorem] states:

Theorem 3.2. If $Y$ is a path-connected space then

$$
\cup \text {-length } Y \leqslant \operatorname{co-nil} \Sigma(Y) \text {. }
$$

Given a space $X$, by Hilton [5, Definition 6.6], w-cat $Y$, the weak category of $Y$ is the least integer $n \geqslant 1$ such that the composition map

$$
Y \stackrel{\Delta}{\longrightarrow} X^{n} \stackrel{q_{n}}{\longrightarrow} Y^{\wedge n}
$$

is nullhomotopic. If no such integer exists, w-cat $Y=\infty$.

Recall that the Lusternik-Schnirelmann category cat $Y$ is the least integer $n \geqslant 1$ such that the space $X$ may be covered by $n$ open subsets which are contractible in $Y$. If no such integer exists, cat $Y=\infty$.

The result [5, Proposition 6.8] states:

Proposition 3.3. If $Y$ is a connected normal well-pointed space then

$$
\text { w-cat } Y \leqslant \operatorname{cat} Y \text {. }
$$

Now, let $\mathcal{H}$ denote the class of all well-pointed $H$-spaces. Certainly, given a space $Y$, the mapping space $X^{Y}$ is an $H$-space for any $X \in \mathcal{H}$. Then, $[5,6.10$. Theorem $]$ states:

Proposition 3.4. If $Y$ is a well-pointed Hausdorff space then

$$
\sup \left\{\text { nil } X^{Y} \mid X \in \mathcal{H}\right\} \leqslant \text { w-cat } Y \text {. }
$$

If $Y$ is a well-pointed space, so does ${ }^{m} Y$ for any $m \geqslant 1$ and $\Omega(Y) \in \mathcal{H}$. It follows from [32, Satz 17 and (33)] that $\Sigma\left({ }^{m} Y\right)$ is also well-pointed and this implies that $\Omega \Sigma^{m}(Y) \in \mathcal{H}$ for any $m \geqslant 1$. Then, the result below stated in $[5,6.11$. Theorem] follows from Theorem 1.7:

Proposition 3.5. If $Y$ is a well-pointed space then

$$
\operatorname{co-nil} \Sigma(Y) \leqslant \sup \{\operatorname{nil}[Y, X] \mid X \in \mathcal{H}\} \text {. }
$$

The result above yields: 
Corollary 3.6. If $Y$ is a path-connected well-pointed normal Hausdorff space then

$\cup$-length $Y \leqslant \sup \{\operatorname{nil}[Y, X] \mid X \in \mathcal{H}\} \leqslant \sup \left\{\operatorname{nil} X^{Y} \mid X \in \mathcal{H}\right\} \leqslant \operatorname{cat} Y-1$.

Furthermore, from Propositions 3.4 and 3.5 we get an upper bound for co-nil $\Sigma(Y)$ :

Corollary 3.7. If $Y$ is a well-pointed Hausdorff space then

$$
\operatorname{co-nil} \Sigma(Y) \leqslant \text { w-cat } Y-1 \leqslant \operatorname{cat} Y-1 \text {. }
$$

Summarizing the above, we get:

Theorem 3.8. If $Y$ is a well-pointed path-connected normal space then

$$
\cup \text {-length } Y \leqslant \operatorname{co-nil} \Sigma(Y) \leqslant \text { w-cat } X-1 \leqslant \operatorname{cat} Y-1 \text {. }
$$

Notice that Theorem 3.8 leads to the following version of Whitehead's [39] result (cf. Lemma 1.4(1)):

Corollary 3.9. If $Y$ is a well-pointed path-connected normal space with cat $Y \leqslant n$, then the nilpotency nil $[Y, X] \leqslant n-1$ for any group-like space $X$.

Proof. Recall that for any group-like space $X$, there is a decomposition via $H$-maps (up to homotopy), of the identity map $\iota_{X}$ as $X \rightarrow \Omega \Sigma(X) \rightarrow X$. Hence, we may consider the group $[Y, X]$ as a subgroup of $[Y, \Omega \Sigma(X)]$. From Theorem 3.8, we deduce that the nilpotency of $[Y, X]$ is less than or equal to co-nil $\Sigma(Y) \leqslant$ cat $Y-1=n-1$ and the proof follows.

In particular, we get that nil $[Y, \Omega(X)]=\operatorname{nil}[\Sigma(Y), X] \leqslant n-1$ for any spaces $X, Y$ provided $Y$ is a well-pointed 0 -connected normal space with cat $Y \leqslant n$.

We conclude this section by giving some examples.

Example 3.10. (1) co-nil $\mathbb{S}^{n}=1$ since the sphere $\mathbb{S}^{n}$ is co-commutative co- $H$-space provided $n \geqslant 2$.

(2) If $A$ is an Abelian group and $M(A, n)$ the Moore space of type $(A, n)$ with $n \geqslant 3$ then $M(A, n) \simeq \Sigma^{2}(M(A, n-2))$. Hence, co-nil $M(A, n)=1$.

If $A$ is a finitely generated then

$$
A \approx \mathbb{Z}^{k} \oplus \mathbb{Z}_{m_{1}} \oplus \cdots \oplus \mathbb{Z}_{m_{l}}
$$

for some $k, l \geqslant 0$ with $\mathbb{Z}_{m}$ the cyclic group of order $m$. Then the Moore space

$$
M(A, 2) \simeq{ }^{k}\left(\mathbb{S}^{n}\right) \vee M\left(\mathbb{Z}_{m_{1}}, 2\right) \vee \cdots \vee M\left(\mathbb{Z}_{m_{l}}, 2\right)
$$


and cat $M(A, 2)<\infty$ implies, by means of Corollary 3.7, that

$$
\text { co-nil } M(A, 2) \leqslant \operatorname{cat} M(A, 2)-1<\infty .
$$

In view of [3, Proposition 13], the space $M\left(\mathbb{Z}_{m}, 2\right)$ admits $m$ distinct co$H$-structures and by $\left[3\right.$, Corollary 16], any two co- $H$-structures on $M\left(\mathbb{Z}_{m}, 2\right)$ are equivalent. Consequently, all co- $H$-structures on $M\left(\mathbb{Z}_{m}, 2\right)$ are associative. If $m$ is odd, all co- $H$-structures on $M\left(\mathbb{Z}_{m}, 2\right)$ are co-commutative and so

$$
\text { co-nil } M\left(\mathbb{Z}_{m}, 2\right)=1 .
$$

If $m$ is even, no co- $H$-structures on $M\left(\mathbb{Z}_{m}, 2\right)$ is co-commutative. But, $M\left(\mathbb{Z}_{m}, 2\right)=\Sigma\left(\mathbb{S}^{1} \cup_{m} \mathbb{S}^{1}\right)$ with $m: \mathbb{S}^{1} \rightarrow \mathbb{S}^{1}$ the map of degree $m$. Since cat $\left(\mathbb{S}^{1} \cup_{m} \mathbb{S}^{1}\right)=3$, by means of Corollary 3.7 , we get that

$$
\text { co-nil } M\left(\mathbb{Z}_{m}, 2\right)=2 \text {. }
$$

\section{The HOMOtopy NiLPotency OF SOME HOMOGENEOUS SPACES}

We make mainly use of [16] to review the homotopy nilpotency $\Omega(G / K)$ of loop spaces for some Lie groups $G$ and their closed subgroups $K<G$.

The homotopy nilpotency of $H$-spaces has been extensively studied as well as the homotopy commutativity. The first major advance was made by Hopkins [22, Theorem 2.1] and completed by Rao [34, Theorem 0.2]. He showed that a finite $H$-space $X$ is homotopy nilpotent if and only if for sufficiently large $n, \varphi_{X, n}$ 's induce trivial homomorphism in complex bordism. This is the same as asking that $\varphi_{X, n}$ 's induce trivial homomorphisms in all Morava $K$-theories. We point out that [22, Theorem 2.1] has been proved by reducing the problem to one in stable homotopy theory and applying the nilpotence theorem [11]. Then, in [22, Corollary 2.2], it was deduced:

Corollary 4.1. If $X$ is a finite associative $H$-space and the homology $H_{*}(X, \mathbb{Z})$ is torsion free then $X$ is homotopy nilpotent.

This corollary implies:

$$
\operatorname{nil} \mathrm{U}(n)<\infty \quad \text { and } \quad \operatorname{nil} \operatorname{Sp}(n)<\infty .
$$

Because of $H$-homotopy equivalences

$$
\mathrm{O}(n) \simeq \mathrm{SO}(n) \times \mathbb{Z}_{2} \quad \text { and } \quad \mathrm{U}(n) \simeq \mathrm{SU}(n) \times \mathbb{S}^{1},
$$

we derive:

$$
\operatorname{nil} \mathrm{SO}(n)=\operatorname{nil} \mathrm{O}(n) \quad \text { and } \quad \operatorname{nilSU}(n)=\operatorname{nil} \mathrm{U}(n)<\infty .
$$

Next, write

$$
\mathrm{O}=\lim _{\rightarrow} \mathrm{O}(n), \quad \mathrm{U}=\lim _{\rightarrow} \mathrm{U}(n), \quad \mathrm{Sp}=\lim _{\rightarrow} \mathrm{Sp}(n) .
$$


Then, notice that by Bott periodicity theorem:

$$
\Omega^{8}(\mathrm{O}) \simeq \mathrm{O}, \quad \Omega^{2}(\mathrm{U}) \simeq \mathrm{U}, \quad \Omega^{8}(\mathrm{Sp}) \simeq \mathrm{Sp},
$$

we get

$$
\operatorname{nil} \mathrm{O}=\operatorname{nil} \mathrm{U}=\operatorname{nil} \mathrm{Sp}=1 .
$$

But, the classical homotopy nilpotency does not capture the algebraic nilpotency of a topological group.

Remark 4.2. Since the commutators

$$
[\mathrm{SO}(3), \mathrm{SO}(3)]=\mathrm{SO}(3) \quad \text { and } \quad[\mathrm{SU}(2), \mathrm{SU}(2)]=\mathrm{SU}(2)
$$

and $\mathrm{SO}(3) \subseteq \mathrm{SO}(n) \subseteq \mathrm{O}(n)$ for $n \geqslant 3, \mathrm{SU}(2) \subseteq \mathrm{SU}(n) \subseteq \mathrm{U}(n)$ for $n \geqslant 2$ and $\mathrm{SU}(2)=\mathrm{Sp}(1) \subseteq \mathrm{Sp}(n)$ for $n \geqslant 1$, we derive that the groups:

(1) $\mathrm{SO}(n)$ and $\mathrm{O}(n)$ are not nilpotent for $n \geqslant 3$;

(2) $\mathrm{U}(n)$ and $\mathrm{SU}(n)$ are not nilpotent for $n \geqslant 2$;

(3) $\operatorname{Sp}(n)$ is not nilpotent for $n \geqslant 1$.

Recall that Biedermann and Dwyer setting [7] define homotopy $n$-nilpotent groups as homotopy algebras over certain simplicial algebraic theories to study the connection between the Goodwillie tower of the identity and the lower central series of the loop group on connected spaces. This notion interpolates between infinite loop spaces and loop spaces, but backwards. They study the relation to ordinary nilpotent groups and prove that $n$ excisive functors of the form $\Omega(F)$ factor over the category of homotopy $n$-nilpotent groups.

Nilpotency for discrete groups can be defined in terms of central extensions. Costoya, Scherer, and Viruel [9] study the analogous definition for spaces in terms of principal fibrations having infinite loop spaces as fibers, yielding a new invariant they compare with classical co-category, but also with the more recent notion of homotopy nilpotency introduced by Biedermann and Dwyer [7]. This allows to characterize finite homotopy nilpotent loop spaces in the spirit of Hubbuck's Torus Theorem and corresponding results for $p$-compact groups and $p$-Noetherian groups.

Later, Rao [33] showed that the converse of the criterion from Corollary 4.1 is true in the case of $\operatorname{Spin}(n)$ and $\mathrm{SO}(n)$ by showing that $\operatorname{Spin}(n)$, $\mathrm{SO}(n), n \geqslant 7$ and $\mathrm{SO}(3), \mathrm{SO}(4)$ are not homotopy nilpotent.

Let now $G_{(p)}$ stand for the $p$-localization in the sense of [8] of a compact Lie group $G$. Then, Yagita [40, Theorem] has shown:

Theorem 4.3. Let $G$ be a simply connected Lie group. Then for each prime $p$, the p-localization $G_{(p)}$ is homotopy nilpotent if and only if the integral cohomology $H^{*}(G, \mathbb{Z})$ has no p-torsion. 
Next, Rao [34, Theorem 0.2] has generalized Theorem 4.3 as follows:

Theorem 4.4. Let $G$ be a compact connected Lie group and let $p$ be a prime. Then $G_{(p)}$ is homotopy nilpotent if and only if $H_{*}\left(G, \mathbb{Z}_{(p)}\right)$ is torsion-free.

Recall that an $H$-map $f: X \rightarrow Y$ of $H$-spaces is called central provided

$$
\bar{\varphi}_{2, Y}\left(f \wedge \iota_{Y}\right) \simeq * \text {. }
$$

Then, in view of [42, Lemma 2.6.6], we have:

Lemma 4.5. Let $F \stackrel{i}{\rightarrow} E \stackrel{q}{\rightarrow} B$ be an $H$-fibration, i.e., $F \stackrel{i}{\rightarrow} E \stackrel{q}{\rightarrow} B$ is a fibration, $F, E$ and $B$ are $H$-spaces and the maps $i: F \rightarrow E$, and $q: E \rightarrow B$ are $H$-maps.

(1) If nil $q \leqslant n$ and $i: F \rightarrow E$ is central then nil $E \leqslant n+1$.

(2) If $\Omega(Y) \stackrel{i}{\rightarrow} E \stackrel{q}{\rightarrow} X$ is the induced $H$-fibration by an $H$-map $f: X \rightarrow Y$ then the map $i: \Omega(Y) \rightarrow E$ is central.

If a topological group $G$ acts freely on a paracompact space $X$ then there is a homeomorphism

$$
X / G \approx X \times_{G} E G,
$$

where $G \rightarrow E G \rightarrow B G$ is the universal principal $G$-bundle.

Since the connecting map $\partial_{X}: \Omega(X / G) \rightarrow G$, in view of [36, Theorem 8.6] (see also [18, Corollary 3.4]), is an $H$-map, the fibration

$$
X \rightarrow X / G \rightarrow E G / G \approx B G
$$

leads to the $H$-fibration

$$
\Omega(X) \longrightarrow \Omega(X / G) \stackrel{\partial_{X}}{\longrightarrow} G .
$$

Now, let $G$ be a compact Lie group and $K<G$ be its closed subgroup. Then the quotient $G / K$ is a manifold and the quotient map $q: G \rightarrow G / K$ is a submersion. Hence, $q: G \rightarrow G / K$ has a local section at the point $q(e)=K$ for the unit $e \in G$. This certainly implies that $q: G \rightarrow G / K$ has a local section at any point $q(g)$ for any $g \in G$. Consequently, the quotient map $q: G \rightarrow G / K$ is a fiber bundle with the fiber $K$ as a principal $H$ bundle. Thus, we have an $H$-fibration

$$
\Omega(G) \longrightarrow \Omega(G / K) \stackrel{\partial_{G}}{\longrightarrow} K .
$$

Since this fibration is induced by the inclusion map $K \hookrightarrow G$ which is certainly an $H$-map, Lemma 4.5 yields:

Proposition 4.6. If $G$ is a compact Lie group and $K<G$ its closed subgroup with nil $K<\infty$ then nil $\Omega(G / K)<\infty$. 
Grassmannians. Let $\mathbb{K}=\mathbb{R}, \mathbb{C}$ be the field of reals or complex numbers and $\mathbb{H}$, the skew $\mathbb{R}$-algebra of quaternions. Then, we set:

$$
\mathrm{U}_{\mathbb{K}}(n)=\left\{\begin{array}{ll}
\mathrm{O}(n) & \text { if } \mathbb{K}=\mathbb{R}, \\
\mathrm{U}(n) & \text { if } \mathbb{K}=\mathbb{C}, \\
\mathrm{Sp}(n) & \text { if } \mathbb{K}=\mathbb{H},
\end{array} \quad \mathrm{SU}_{\mathbb{K}}(n)= \begin{cases}\mathrm{SO}(n) & \text { if } \mathbb{K}=\mathbb{R} \\
\mathrm{SU}(n) & \text { if } \mathbb{K}=\mathbb{C} \\
\mathrm{Sp}(n) & \text { if } \mathbb{K}=\mathbb{H}\end{cases}\right.
$$

Write $G_{n, m}(\mathbb{K})$ (resp. $\quad G_{n, m}^{+}(\mathbb{K})$ ) for the (resp. oriented) Grassmannian of $m$-dimensional subspaces in the $n$-dimensional $\mathbb{K}$-vector space. For example, the set of lines $G_{n+1,1}(\mathbb{K})=\mathbb{K} P^{n}$ is the projective $n$-space over $\mathbb{K}$.

The homotopy nilpotency of the loop spaces $\Omega\left(\mathbb{K} P^{n}\right)$ has been first studied by Ganea [13], Snaith [35] and then their $p$-localization $\Omega\left(\left(\mathbb{K} P^{n}\right)_{(p)}\right)$ by Meier [28]. Recall that by Ganea [13, Propositions 1.3-1.5] and Snaith [35, Corollaries 2.6 and 2.13], we have:

Proposition 4.7. The following statements hold:

(1) $\operatorname{nil} \Omega\left(\mathbb{R} P^{2 n+1}\right)= \begin{cases}\leqslant 2, & \text { for } n \geqslant 0, \\ 1, & \text { if and only if } n=0,1 \text { or } 3\end{cases}$

(2) $\operatorname{nil} \Omega\left(\mathbb{R} P^{2 n}\right)=\infty$ for $n \geqslant 1$;

(3) nil $\Omega\left(\mathbb{C} P^{2 n+1}\right)= \begin{cases}\leqslant 2, & \text { for any odd } n \geqslant 1, \\ 1, & \text { if and only if } n=1 ;\end{cases}$

(4) $4 \leqslant \operatorname{nil} \Omega\left(\mathbb{C} P^{2 n}\right) \leqslant 6$ for $n \geqslant 1$;

(5) $3 \leqslant \operatorname{nil} \Omega\left(\mathbb{H} P^{n}\right)$ for any $n \geqslant 1$;

(6) $\operatorname{nil} \Omega\left(\mathbb{H} P^{n}\right)=3$ if $n \equiv-1(\bmod 24)$.

Then, Meier [28, Theorem 5.4] has shown some results on the homotopy nilpotency of $p$-localized projective spaces:

Theorem 4.8. Let $p$ be an odd prime and $n \geqslant 2$ a natural number. Then:

(1) $\operatorname{nil} \Omega\left(\mathbb{C} P_{(p)}^{n}\right)=1$;

(2) $\operatorname{nil} \Omega\left(\mathbb{H} P_{(p)}^{n}\right)=1$ if $p>3$;

(3) $3 \leqslant \operatorname{nil} \Omega\left(\mathbb{H} P_{(3)}^{n}\right) \leqslant 4$;

(4) $\operatorname{nil} \Omega\left(\mathbb{H} P_{(3)}^{n}\right)=3$ if $n \equiv 2(\bmod 3)$.

Since the space $\mathbb{R} P^{2 n+1}$ is simple, there is its localization $\mathbb{R} P_{(p)}^{2 n+1}$ for any prime $p \geqslant 2$. It it also easy to see that nil $\Omega\left(\mathbb{R} P_{(p)}^{2 n+1}\right)=1$ for any odd 
prime $p$ and $n \geqslant 0$. But, the nilpotency nil $\Omega\left(\mathbb{H} P^{n}\right)$ for any $n \geqslant 2$ does not appear in the literature known to the author.

It is well known that $G_{n, m}(\mathbb{K})$ (resp. $G_{n, m}^{+}(\mathbb{K})$ ) are smooth manifolds with diffeomorphisms

$$
\begin{aligned}
& G_{n, m}(\mathbb{K}) \approx \mathrm{U}_{\mathbb{K}}(n) /\left(\mathrm{U}_{\mathbb{K}}(m) \times \mathrm{U}_{\mathbb{K}}(n-m)\right), \\
& G_{n, m}^{+}(\mathbb{K}) \approx \mathrm{SU}_{\mathbb{K}}(n) /\left(\mathrm{SU}_{\mathbb{K}}(m) \times \mathrm{SU}_{\mathbb{K}}(n-m)\right)
\end{aligned}
$$

for $\mathbb{K}=\mathbb{R}, \mathbb{C}, \mathbb{H}$.

Since the homomorphism $\pi_{1}\left(\mathrm{SU}(m)_{\mathbb{K}}\right) \rightarrow \pi_{1}\left(\mathrm{SU}(n)_{\mathbb{K}}\right)$ of fundamental groups determined by the inclusion map $\mathrm{SU}(m)_{\mathbb{K}} \hookrightarrow \mathrm{SU}(n)_{\mathbb{K}}$ for $2 \leqslant m \leqslant n$ is an epimorphism, we derive that the spaces $G_{n, m}^{+}(\mathbb{K})$ are simply connected for $\mathbb{K}=\mathbb{R}, \mathbb{C}, \mathbb{H}$. Next, there is the universal covering map

$$
\mathbb{Z}_{2} \longrightarrow G_{n, m}^{+}(\mathbb{R}) \longrightarrow G_{n, m}(\mathbb{R})
$$

and a fibre bundle

$$
\mathbb{S}^{1} \longrightarrow G_{n, m}^{+}(\mathbb{C}) \longrightarrow G_{n, m}(\mathbb{C}) .
$$

Now, recall that the classifying

$$
\mathrm{BU}_{\mathbb{K}}(m)=\lim _{\rightarrow} G_{n, m}(\mathbb{K})=G_{\infty, m}(\mathbb{K}) .
$$

Since the cohomology $H^{*}(\mathrm{SO}(n), \mathbb{Z})$ has only 2-torsion and $H^{*}(U(n), \mathbb{Z})$, $H^{*}(\operatorname{Sp}(n), \mathbb{Z})$ are torsion free, the fibration

$$
\Omega\left(\mathrm{SU}_{\mathbb{K}}(n)\right) \longrightarrow \Omega\left(G_{n, m}^{+}(\mathbb{K})\right) \longrightarrow \mathrm{SU}_{\mathbb{K}}(m) \times \mathrm{SU}_{\mathbb{K}}(n-m),
$$

Corollary 4.1, Theorem 4.4 and Proposition 4.6 lead to:

Proposition 4.9. If $1 \leqslant m<n \leqslant \infty$ then:

(1) $\operatorname{nil} \Omega\left(G_{n, m}^{+}(\mathbb{R})_{(p)}\right)<\infty$ for $p>2$;

$(2) \operatorname{nil} \Omega\left(G_{n, m}(\mathbb{K})\right)<\infty$ and nil $\Omega\left(G_{n, m}^{+}(\mathbb{K})\right)<\infty$ for $\mathbb{K}=\mathbb{C}, \mathbb{H}$.

In particular, nil $\Omega\left(\mathbb{H} P^{n}\right)<\infty$.

We do not mention above any result on the $p$-localization of $G_{n, m}(\mathbb{R})$ because we are not sure on its existence.

Remark 4.10. The (resp. oriented) flag manifold $F_{n ; n_{1}, \ldots, n_{k}}(\mathbb{K})$ (resp. $\left.F_{n ; n_{1}, \ldots, n_{k}}^{+}(\mathbb{K})\right)$ with $1 \leqslant n_{1}<\cdots<n_{k} \leqslant n-1$ in the $n$-dimensional $\mathbb{K}$-vector space is smooth with a diffeomorphism

$$
F_{n ; n_{1}, \ldots, n_{k}}(\mathbb{K}) \approx \frac{\mathrm{U}_{\mathbb{K}}(n)}{\mathrm{U}_{\mathbb{K}}\left(n_{1}\right) \times \mathrm{U}_{\mathbb{K}}\left(n_{1}-n_{2}\right) \times \cdots \times \mathrm{U}_{\mathbb{K}}\left(n_{k-1}-n_{k}\right) \times \mathrm{U}_{\mathbb{K}}\left(n-n_{k}\right)},
$$

resp.

$$
F_{n ; n_{1}, \ldots, n_{k}}^{+}(\mathbb{K}) \approx \frac{\operatorname{SU}_{\mathbb{K}}(n)}{\left.\operatorname{SU}_{\mathbb{K}}\left(n_{1}\right) \times \mathrm{SU}_{\mathbb{K}}\left(n_{1}-n_{2}\right) \times \cdots \times \mathrm{SU}_{\mathbb{K}}\left(n_{k-1}-n_{k}\right) \times \mathrm{SU}_{\mathbb{K}}\left(n-n_{k}\right)\right)} .
$$


Furthermore, there is the universal covering map

$$
\left(\mathbb{Z}_{2}\right)^{k} \rightarrow F_{n ; n_{1}, \ldots, n_{k}}^{+}(\mathbb{R}) \rightarrow F_{n ; n_{1}, \ldots, n_{k}}(\mathbb{R})
$$

and a fibre bundle

$$
\left(\mathbb{S}^{1}\right)^{k} \rightarrow F_{n ; n_{1}, \ldots, n_{k}}^{+}(\mathbb{C}) \rightarrow F_{n ; n_{1}, \ldots, n_{k}}(\mathbb{C}) .
$$

Consequently, Corollary 4.1, Theorem 4.4, and Proposition 4.6 lead to:

(1) $\operatorname{nil} \Omega\left(F_{n ; n_{1}, \ldots, n_{k}}^{+}(\mathbb{R})_{(p)}\right)<\infty$ for $p>2$;

(2) nil $\Omega\left(F_{n ; n_{1}, \ldots, n_{k}}(\mathbb{K})\right)<\infty$ and nil $\Omega\left(F_{n ; n_{1}, \ldots, n_{k}}^{+}(\mathbb{K})\right)<\infty$ for $\mathbb{K}=\mathbb{C}, \mathbb{H}$.

As above, we do not mention above any result on the $p$-localization of $F_{n ; n_{1}, \ldots, n_{k}}(\mathbb{R})$ because we are not sure on its existence.

Stiefel manifolds. The Stiefel manifold $V_{n, m}(\mathbb{K})$ is the set of all orthonormal $m$-frames in the vector space $\mathbb{K}^{n}$. That is, it is the set of ordered orthonormal $m$-tuples of vectors in $\mathbb{K}^{n}$ for $\mathbb{K}=\mathbb{R}, \mathbb{C}$ or $\mathbb{H}$.

It is well known that $V_{n, m}(\mathbb{K})$ is a smooth manifold and there are diffeomorphisms:

(1) $V_{n, m}(\mathbb{R})=V_{n, m} \approx \mathrm{O}(n) / \mathrm{O}(n-m) \approx \mathrm{SO}(n) / \mathrm{SO}(n-m)$;

(2) $V_{n, m}(\mathbb{C})=W_{n, m} \approx \mathrm{U}(n) / \mathrm{U}(n-m) \approx \mathrm{SU}(n) / \mathrm{SU}(n-m)$;

(3) $V_{n, m}(\mathbb{H})=X_{n, m} \approx \operatorname{Sp}(n) / \operatorname{Sp}(n-m)$.

Since the homomorphism $\pi_{1}\left(\mathrm{SU}(m)_{\mathbb{K}}\right) \rightarrow \pi_{1}\left(\mathrm{SU}(n)_{\mathbb{K}}\right)$ of fundamental groups determined by the inclusion map $\mathrm{SU}(m)_{\mathbb{K}} \hookrightarrow \mathrm{SU}(n)_{\mathbb{K}}$ for $2 \leqslant m \leqslant n$ is an epimorphism, we derive that the spaces $V_{n, m}(\mathbb{K})$ are simply connected for $\mathbb{K}=\mathbb{R}, \mathbb{C}, \mathbb{H}$. Then, Corollaries 4.1 and 4.6 lead to:

Proposition 4.11. If $1 \leqslant m \leqslant n$ then:

(1) $\operatorname{nil} \Omega\left(V_{n, m}\right)_{(p)}<\infty$ for $p>2$;

(2) $\operatorname{nil} \Omega\left(W_{n, m}\right)<\infty$;

(3) $\operatorname{nil} \Omega\left(X_{n, m}\right)<\infty$.

Acknowledgments. The author greatly appreciate the careful reading of the manuscript, the thoughtful comments and suggestions of the anonymous referee.

\section{REFERENCES}

[1] Martin Arkowitz. Induced mappings of homology decompositions. In Homotopy and geometry (Warsaw, 1997), volume 45 of Banach Center Publ., pages 225-233. Polish Acad. Sci. Inst. Math., Warsaw, 1998, https://eudml.org/doc/208905.

[2] Martin Arkowitz. Introduction to homotopy theory. Universitext. Springer, New York, 2011, doi: 10.1007/978-1-4419-7329-0. 
[3] Martin Arkowitz, Marek Golasiński. Co- $H$-structures on Moore spaces of type $(G, 2)$. Canad. J. Math., 46(4):673-686, 1994, doi: 10.4153/CJM-1994-037-0.

[4] Clemens Berger, Dominique Bourn. Central reflections and nilpotency in exact Mal'tsev categories. J. Homotopy Relat. Struct., 12(4):765-835, 2017, doi: 10.1007/s40062-0160165-8.

[5] I. Berstein, T. Ganea. Homotopical nilpotency. Illinois J. Math., 5:99-130, 1961, doi: $10.1215 / \mathrm{ijm} / 1255629648$.

[6] Israel Berstein, Emmanuel Dror. On the homotopy type of non-simply-connected co$H$-spaces. Illinois J. Math., 20(3):528-534, 1976, doi: 10.1215/ijm/1256049794.

[7] Georg Biedermann, William G. Dwyer. Homotopy nilpotent groups. Algebr. Geom. Topol., 10(1):33-61, 2010, doi: 10.2140/agt.2010.10.33.

[8] A. K. Bousfield, D. M. Kan. Homotopy limits, completions and localizations. Lecture Notes in Mathematics, Vol. 304. Springer-Verlag, Berlin-New York, 1972, doi: 10.1007/978-3-540-38117-4.

[9] Cristina Costoya, Jérôme Scherer, Antonio Viruel. A torus theorem for homotopy nilpotent loop spaces. Ark. Mat., 56(1):53-71, 2018, doi: 10.4310/ARKIV.2018.v56.n1.a5.

[10] M. C. Crabb, W. A. Sutherland, P. Zhang. Homotopy nilpotency. Quart. J. Math. Oxford Ser. (2), 50(198):179-196, 1999, doi: 10.1093/qjmath/50.198.179.

[11] Ethan S. Devinatz, Michael J. Hopkins, Jeffrey H. Smith. Nilpotence and stable homotopy theory. I. Ann. of Math. (2), 128(2):207-241, 1988, doi: 10.2307/1971440.

[12] Rosona Eldred. Goodwillie calculus via adjunction and LS cocategory. Homology Homotopy Appl., 18(2):31-58, 2016, doi: 10.4310/HHA.2016.v18.n2.a2.

[13] T. Ganea. On the loop spaces of projective spaces. J. Math. Mech., 16:853-858, 1967, doi: $10.2307 / 45277192$.

[14] Tudor Ganea. Lusternik-Schnirelmann category and cocategory. Proc. London Math. Soc. (3), 10:623-639, 1960, doi: 10.1112/plms/s3-10.1.623.

[15] Tudor Ganea. Cogroups and suspensions. Invent. Math., 9:185-197, 1969/70, doi: 10.1007/BF01404323.

[16] M. Golasiński. Homotopy nilpotency of some homogeneous spaces. Submitted, 2020.

[17] M. Golasiński. On two equivalent notions of the homotopy nilpotency. Submitted, 2020.

[18] Marek Golasiński, Daciberg Lima Gonçalves, Peter Wong. Exponents of $\left[\Omega\left(\mathbb{S}^{r+1}\right), \Omega(Y)\right]$. In Algebraic topology and related topics, Trends Math., pages 103-122. Birkhäuser/Springer, Singapore, 2019, doi: 10.1007/978-981-13-5742-8_ 7.

[19] Marek Golasiński, John R. Klein. On maps into a co- $H$-space. Hiroshima Math. J., 28(2):321-327, 1998, doi: 10.32917/hmj/1206126763.

[20] Peter Hilton. Homotopy theory and duality. Gordon and Breach Science Publishers, New York-London-Paris, 1965.

[21] M. J. Hopkins. Formulations of cocategory and the iterated suspension. 113:212-226, 1984, http://www.numdam.org/item/AST_1984__113-114__212_0.

[22] M. J. Hopkins. Nilpotence and finite $H$-spaces. Israel J. Math., 66(1-3):238-246, 1989, doi: $10.1007 / \mathrm{BF} 02765895$.

[23] M. Hovey. Lusternik-Schnirelmann cocategory. Illinois J. Math., 37(2):224-239, 1993, doi: $10.1215 / \mathrm{ijm} / 1255987145$.

[24] I. M. James. On fibre spaces and nilpotency. II. Math. Proc. Cambridge Philos. Soc., 86(2):215-217, 1979, doi: 10.1017/S0305004100056024.

[25] Donald W. Kahn. A note on $H$-spaces and Postnikov systems of spheres. Proc. Amer. Math. Soc., 15:300-307, 1964, doi: 10.2307/2034058.

[26] Shizuo Kaji, Daisuke Kishimoto. Homotopy nilpotency in p-regular loop spaces. Math. Z., 264(1):209-224, 2010, doi: 10.1007/s00209-008-0459-6. 
[27] Daisuke Kishimoto. Homotopy nilpotency in localized SU(n). Homology Homotopy Appl., 11(1):61-79, 2009, http://projecteuclid.org/euclid.hha/1251832560.

[28] Willi Meier. Homotopy nilpotency and localization. Math. Z., 161(2):169-183, 1978, doi: 10.1007/BF01214929.

[29] Aniceto Murillo, Antonio Viruel. Lusternik-Schnirelmann cocategory: a Whitehead dual approach. In Cohomological methods in homotopy theory (Bellaterra, 1998), volume 196 of Progr. Math., pages 323-347. Birkhäuser, Basel, 2001, doi: 10.1007/978-30348-8312-2 20.

[30] G. J. Porter. Homotopical nilpotence of $S^{3}$. Proc. Amer. Math. Soc., 15:681-682, 1964, doi: $10.2307 / 2034577$.

[31] G. J. Porter. Higher-order Whitehead products. Topology, 3:123-135, 1965, doi: 10.1016/0040-9383(65)90039-X.

[32] Dieter Puppe. Homotopiemengen und ihre induzierten Abbildungen. I. Math. Z., 69:299-344, 1958, doi: 10.1007/BF01187411.

[33] Vidhyanath K. Rao. $\operatorname{Spin}(n)$ is not homotopy nilpotent for $n \geqslant 7$. Topology, 32(2):239249, 1993, doi: 10.1016/0040-9383(93)90017-P.

[34] Vidhyānāth K. Rao. Homotopy nilpotent Lie groups have no torsion in homology. Manuscripta Math., 92(4):455-462, 1997, doi: 10.1007/BF02678205.

[35] Victor P. Snaith. Some nilpotent $H$-spaces. Osaka Math. J., 13(1):145-156, 1976, http: //projecteuclid.org/euclid.ojm/1200769310.

[36] James Stasheff. H-spaces from a homotopy point of view. Lecture Notes in Mathematics, Vol. 161. Springer-Verlag, Berlin-New York, 1970, doi: 10.1007/BFb0065896.

[37] Stephen Theriault. The dual polyhedral product, cocategory and nilpotence. Adv. Math., 340:138-192, 2018, doi: 10.1016/j.aim.2018.09.037.

[38] George W. Whitehead. On products in homotopy groups. Ann. of Math (2), 47:460475, 1946, doi: 10.2307/1969085.

[39] George W. Whitehead. On mappings into group-like spaces. Comment. Math. Helv., 28:320-328, 1954, doi: 10.1007/BF02566938.

[40] Nobuaki Yagita. Homotopy nilpotency for simply connected Lie groups. Bull. London Math. Soc., 25(5):481-486, 1993, doi: 10.1112/blms/25.5.481.

[41] Donald Yau. Clapp-Puppe type Lusternik-Schnirelmann (co)category in a model category. J. Korean Math. Soc., 39(2):163-191, 2002, doi: 10.4134/JKMS.2002.39.2.163.

[42] Alexander Zabrodsky. Hopf spaces. North-Holland Publishing Co., AmsterdamNew York-Oxford, 1976. North-Holland Mathematics Studies, Vol. 22, Notas de Matemática, No. 59.

Received: January 9, 2020, accepted: July 22, 2020.

Marek Golasiński

Faculty of Mathematics and Computer Science, University of Warmia and Mazury, Seoneczna 54 Street, 10-710 Olsztyn, Poland

Email: marekg@matman.uwm.edu.pl

ORCID: orcid.org/0000-0001-6969-8986 